\title{
Fabrication of Patterned Integrated Electrochemical Sensors
}

\author{
Muhammad Mujeeb-U-Rahman, Dvin Adalian, and Axel Scherer \\ California Institute of Technology, Department of Physics and Applied Physics, Pasadena, CA 91125, USA
}

Correspondence should be addressed to Muhammad Mujeeb-U-Rahman; mrahman@caltech.edu

Received 18 March 2015; Revised 15 June 2015; Accepted 25 June 2015

Academic Editor: Carlos R. Cabrera

Copyright (C) 2015 Muhammad Mujeeb-U-Rahman et al. This is an open access article distributed under the Creative Commons Attribution License, which permits unrestricted use, distribution, and reproduction in any medium, provided the original work is properly cited.

\begin{abstract}
Fabrication of integrated electrochemical sensors is an important step towards realizing fully integrated and truly wireless platforms for many local, real-time sensing applications. Micro/nanoscale patterning of small area electrochemical sensor surfaces enhances the sensor performance to overcome the limitations resulting from their small surface area and thus is the key to the successful miniaturization of integrated platforms. We have demonstrated the microfabrication of electrochemical sensors utilizing top-down lithography and etching techniques on silicon and CMOS substrates. This choice of fabrication avoids the need of bottom-up techniques that are not compatible with established methods for fabricating electronics (e.g., CMOS) which form the industrial basis of most integrated microsystems. We present the results of applying microfabricated sensors to various measurement problems, with special attention to their use for continuous DNA and glucose sensing. Our results demonstrate the advantages of using micro- and nanofabrication techniques for the miniaturization and optimization of modern sensing platforms that employ well-established electronic measurement techniques.
\end{abstract}

\section{Introduction}

Ultra-small scale integrated electrochemical sensors have gained considerable interest as solutions to diagnostic monitoring situations requiring a small footprint. One category of microsensors that benefits from miniaturization is medical implants which require fully autonomous sensing platforms along with electronic driving circuitry within the smallest possible volume [1]. Electrochemical sensing technology in particular is a very attractive solution for health monitoring as it is possible to integrate electrochemical circuits with device electronics on a comparatively small size scale [2]. Microscale and nanoscale patterning of the sensor surfaces holds the key to making these devices perform effectively and to reducing the sensor impedance [3]. Such techniques can also be used to enhance the performance of large scale electrodes made via more conventional methods (e.g., screen printing) [4]. Usual implementations of electrochemical sensors commonly utilize a planar electrode configuration, but micro/nanoscale patterning of the electrodes provides many technical advantages such as a higher surface area and the control over the diffusion profile near the electrode surfaces [5]. This surface patterning also allows for more efficient utilization of functional coatings (e.g., enzyme or binding coatings) on the electrodes [6]. Employing these techniques to decrease the total area needed per sensor, along with embedding CMOS electronics underneath the sensor, leads to reducing the system size to practical dimensions [7]. This size reduction also provides major advantages in reducing individual device cost and foreign body response to such devices when used for in vivo applications [8].

The most common fabrication methods of depositing electrodes for electrochemical sensors at small (sub-mm) size scale includes direct Physical Vapor Deposition (PVD) or Chemical Vapor Deposition (CVD). These techniques, when used under nonequilibrium conditions, can yield patterned surfaces [9]. However, the resulting structures are typically not suitable for long-term use because of their deformation in liquid environments as a result of large capillary forces acting on the surface nanostructures in a solution [10]. This limitation can be overcome by use of liquid deposition techniques, for example, VLS growth [11], porous templates [12], or complex electrochemical plating mechanisms [13], since these methods have better resilience to liquid forces. However, such deposition approaches are usually not compatible with the current CMOS fabrication technologies or 
other vacuum-based fabrication processes. Although it has been shown that these alternative methods can be adapted for fabrication on wafer scale, the overall process is generally complex and expensive. Often, it involves deposition of very thick $(10 \mu \mathrm{m})$ metal layers as templates [10]. In the cases that nonvacuum (e.g., liquid) processing has been applied to fabricate nanostructures exact control of the geometry at the nanometer scale has proven difficult. High temperatures, corrosive atmospheres, or nonuniform deposition due to internal stresses during deposition often render such bottomup methods troublesome to scale up with consistency over large areas and over many batches for the reproducible industrial fabrication of low-impedance electrodes [9].

In this work, we present a top-down fabrication methodology to fabricate nanoscale patterns on electrodes with very precise control over the exact geometry and with uniformity over large areas. Our method uses only vacuum-based processing without the need for making electrical contacts to the devices to be patterned or any exotic liquid-based processing. This enables a precise control over the electrochemical environment surrounding the sensor electrodes. Nanofabricated silicon pillars are coated with metal to form our electrodes and exhibit excellent mechanical resilience and successfully resist liquid surface tension forces. We demonstrate deposition of different metals on nanostructures to render them effective for many different sensing and actuation applications. The large and controllable surface area of lithographically patterned surfaces provides a simple and efficient technique to obtain excellent and predictable sensor performance in medical applications such as when quantitatively determining the concentrations of metabolites and when detecting DNA [14]. We believe that this is the first demonstration of metallized nanopillars in fully integrated electrochemical sensor systems with negligible variation in sensor properties over many fabrication batches. Metallization of etched nanostructures can be easily scaled up through industrial fabrication approaches to produce high surface area sensors in existing micro/nanofabrication foundries. Another advantage of metallization of nanostructures is that the sensor elements can be shaped in 3D using well-studied and well-controlled silicon-based fabrication processing. Silicon etching is a very mature and well-understood field, and three-dimensional structures can be achieved with great repeatability as well as geometric accuracy [15].

\section{Device Design}

The sensor design to test the effects of surface area enhancement on electrochemical performance is based upon a three-electrode electrochemical sensor configuration with an optional fourth electrode which can be electrically connected when needed. The standard three electrodes are the working electrode (WE), counter electrode (CE), and reference electrode (RE) [16]. The optional fourth electrode can be used as a second working electrode for differential readings or for background normalization as the sensor ages. All of the electrodes are defined by processing a silicon substrate which allows vacuum-based nanometer scale silicon processing techniques to be applied. The patterned surface benefits from the excellent mechanical elasticity and resilience of nanostructures [17]. The surface material of the electrodes can be chosen to match the requirements of a particular application. Since the sensing mechanism only depends on the exposed surface layer and since most electrochemically active materials are precious metals [18], depositing a thin metal layer on top of an underlying silicon substrate is economically very beneficial. Using only a thin layer also provides mechanical integrity since most of the pillar composition is single crystal silicon. The reference electrode for our electrochemical measurement consists of $\mathrm{Ag} / \mathrm{AgCl}$ thin film bilayer deposited on the RE. The $\mathrm{CE}$ is coated with $\mathrm{Pt}$ for most of our applications and the WE material varies based upon the specific application but is commonly Pt or Au for our devices. A typical sensor is designed to fit in an area of $500 \mu \mathrm{m}$ by $500 \mu \mathrm{m}$ square. The sensor is connected to large contact pads via metal contact lines. The contact lines are insulated (using insulating epoxy, e.g., SU8) to only expose the sensor to test solutions during sensor testing. The contact pads are used to connect the sensor to a test instrument (e.g., Potentiostat) to read its electrical output on a computer. A typical sensor with contact pads is depicted in Figure 1.

The sensor electrodes need to be coated with specific chemistry (i.e., "functionalized") to react with specific molecules for detection in complex biochemical environment [19]. To perform glucose sensing in the presence of many similar molecules in a complex solution, for example, the sensor must be coated with a glucose-sensitive layer [20]. Many different types of functionalization methods have been developed to convert nanoscale sensors into sensitive and specific devices [19]. For amperometric measurements with a redox enzyme, in situ functionalization is a simple and efficient method which allows the application of functionalized materials directly to the sensor surface [21]. This method has the benefit that no electrical connections need to be made to the ultra-small scale devices, which greatly simplifies the fabrication. However, it is important to note that electrochemically deposited coatings can be controlled more precisely and can be used for wired sensors [22].

In this work, functionalization is achieved either by using direct immobilization of a hydrogel containing the desired chemistry (e.g., glucose-sensitive hydrogel) or by wetting the electrodes in a solution containing the desired binding molecules (e.g., nucleic acid strands) depending on the application. A typical sensor geometry employing based functionalization layer is illustrated in Figure 2.

\section{Analysis}

The main reason for micro/nanopatterning is to enhance the sensitivity of the device since it is directly related to the surface area available for the sensing mechanism. This advantage of patterned electrodes over planar electrodes can be quantified by comparing the surface area of a circular pillar patterned electrode $(S)$ as compared to a planar electrode (So) (the derivation for (1) and (2) is given in the Appendix):

$$
\frac{S}{S o}=1+7.26\left(\frac{r}{a}\right)\left(\frac{h}{a}\right) \text {. }
$$




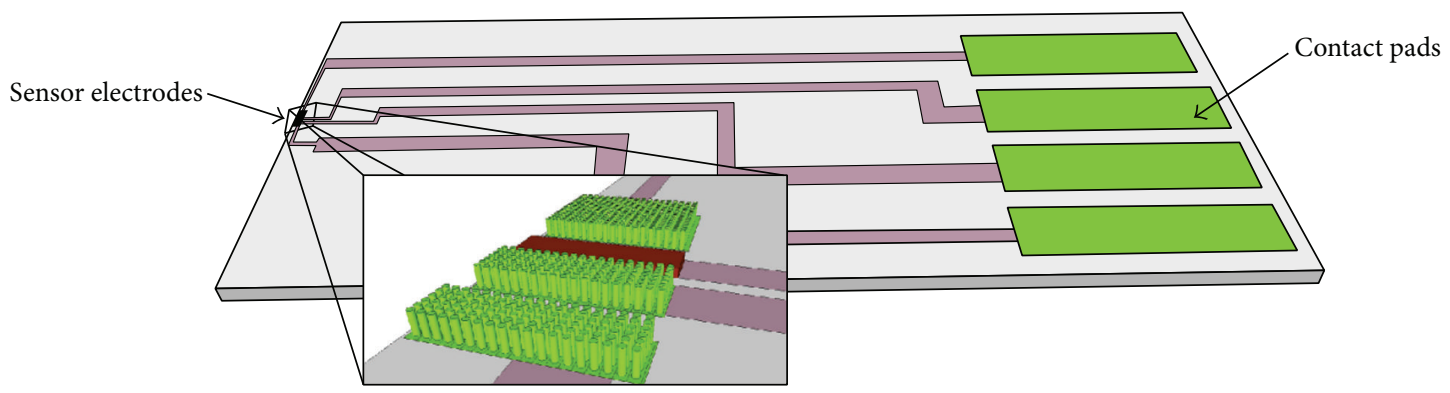

Figure 1: Patterned electrochemical sensor design with contact pads.

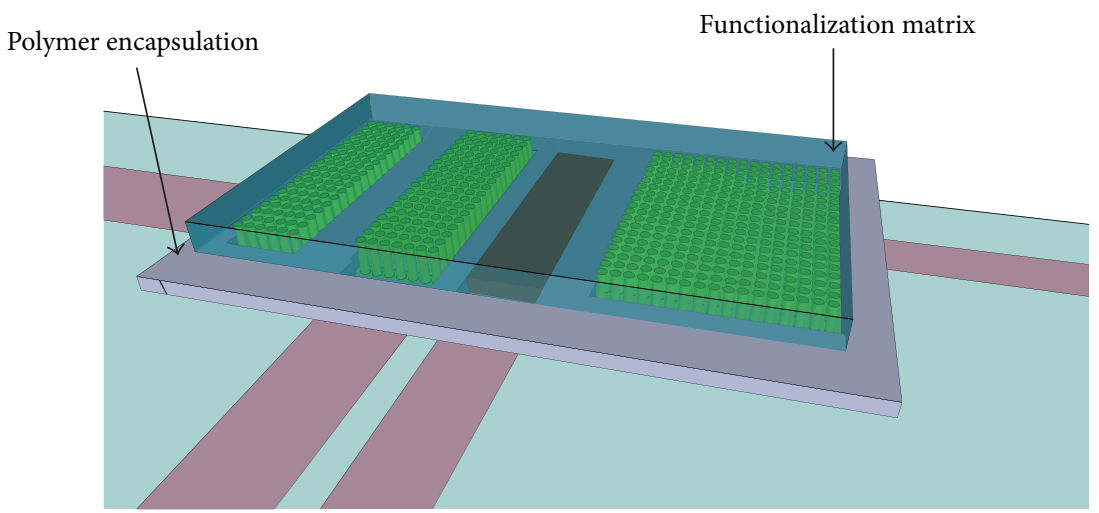

FIgURE 2: Nanopatterned electrodes coated with a functionalizing (immobilization) matrix.

Here, $r$ is the radius of the pillars, $h$ is the height of the pillars, $a$ is the separation between the pillars (center to center distance), and the arrangement is hexagonal closed pack form. For a standard rectangular array of pillars, this surface area equation becomes

$$
\frac{S}{S o}=1+6.28\left(\frac{r}{a}\right)\left(\frac{h}{a}\right) \text {. }
$$

For practical applications, the difference between hexagonal and rectangular packing is negligible, and therefore we utilized rectangular arrangements as these are easier to rescale quickly. The theoretical surface area enhancement from such patterning is plotted against the size of the pillars in Figure 3. Here, pillar aspect ratio is fixed $(h / r=20)$ to signify the effect of reducing pillar radius on surface area.

This shows that higher aspect ratio and higher packing density result in higher surface area for a given geometric area. The exact increase depends upon the scale of patterning and is mostly determined by the application as well as the cost of the micro- or nanofabrication. The smaller the pillar, the more the surface area gain but simultaneously the fabrication becomes more complex and costly. The feature height of the devices is also limited by the surface tension forces in the liquid which can cause sensor damage depending upon both mechanical and geometrical properties of the pillars. The deflection $(\Delta)$ of a pillar due to capillary forces is described by the following equation $[10]$ :

$$
\Delta=\frac{P h^{3}}{3 E I} \text {. }
$$

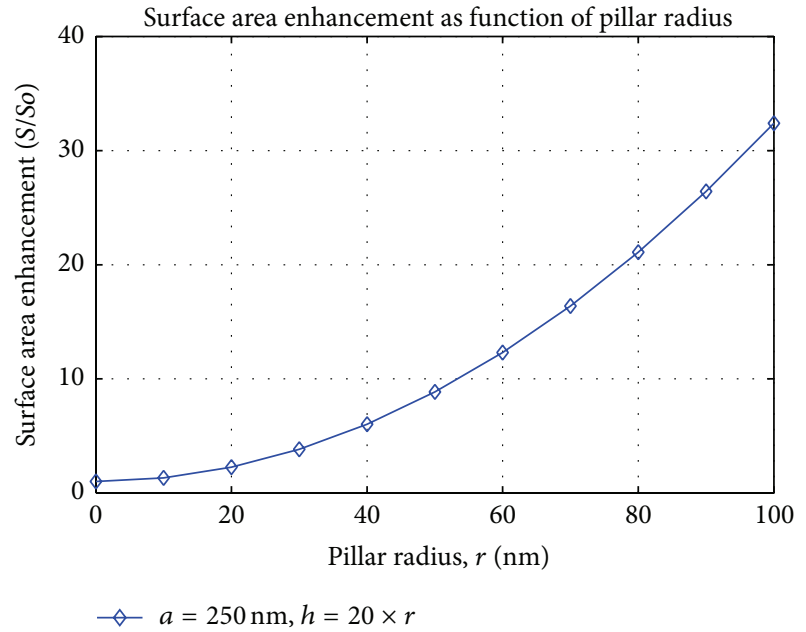

FIGURE 3: Surface area enhancement due to nanopatterning.

Here, $P$ is the capillary force, $h$ is pillar height, $E$ is young's modulus, and $I$ is second moment of inertia given by

$$
I=\frac{\pi r^{4}}{4}
$$

Using (3) and (4),

$$
\Delta=\frac{4 P}{3 \pi E r}\left(\frac{h}{r}\right)^{3}
$$




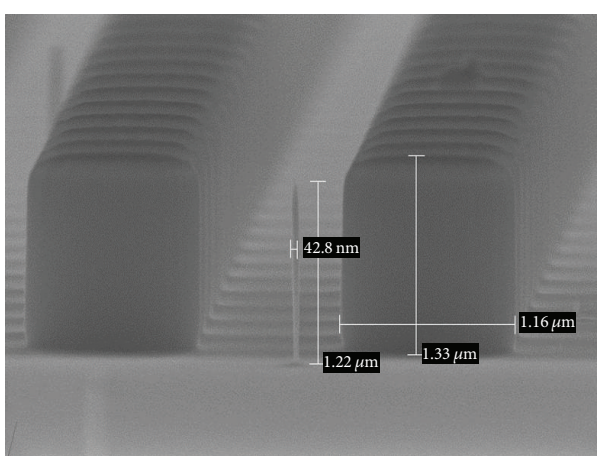

(a)

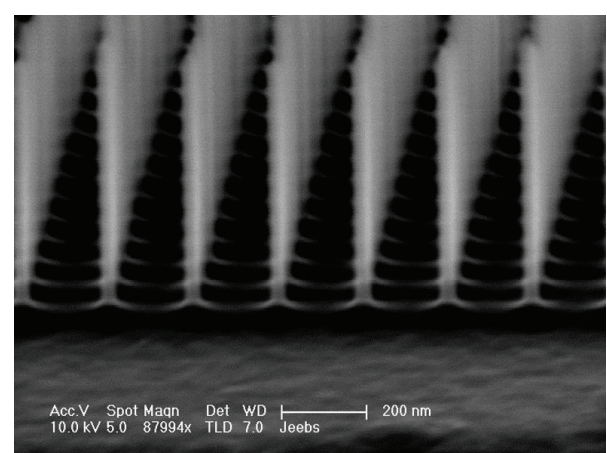

(b)

FIGURE 4: SEM image of plasma-etched nanopillars demonstrating size-independent etching (a) $1 \mu \mathrm{m}$ pillar beside a $50 \mathrm{~nm}$ pillar and (b) $50 \mathrm{~nm}$ pillar array.

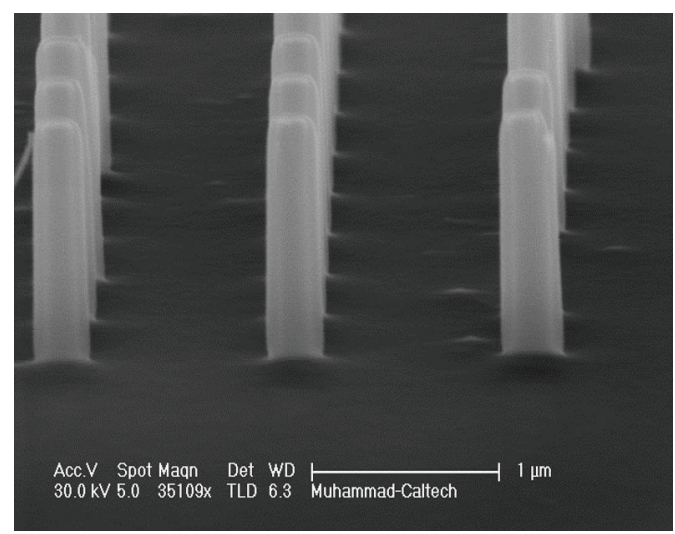

(a)

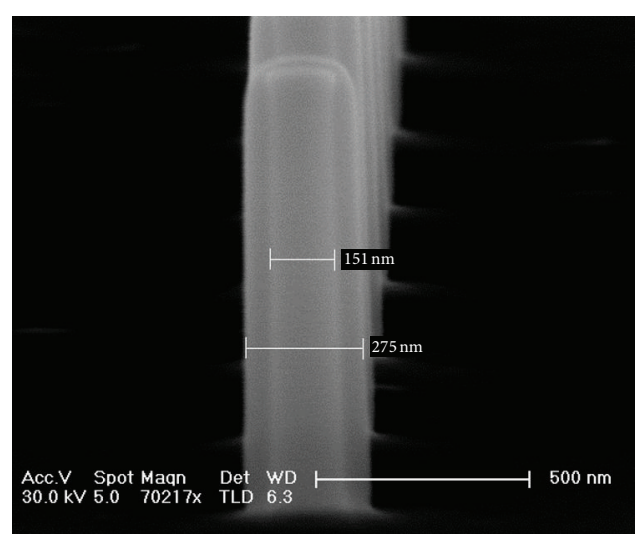

(b)

FIGURE 5: Nanopillars after oxidation. (a) High voltage imaging to confirm the conformality of the oxide layer (b).

This shows that for a given capillary force, surface tension is proportional to third power of pillar aspect ratio $(h / r)$ and is inversely proportional to its Young modulus $(E)$. Control of both of these factors is required to have a safe amount of deflection due to capillary forces and limit sensor damage. Control on young's modulus is achieved by using high purity materials with high Young's modulus. Silicon and Pt group metals have Young's modulus in GPa range in nanostructures and can be appropriately flexible [17]. Aspect ratio control is achieved during fabrication by controlling pattern sizes and etching time, as demonstrated in the next section.

\section{Fabrication}

The complete fabrication sequence used to define our patterned sensor electrodes consists of the following steps: lithographic patterning, pattern transfer processes (e.g., etching), interface control methods including deposition of metals and insulators, isolation coatings between electrodes, and finally, in some applications, functionalization of the metal contact surface.

For nanoscale patterning, EUV or electron beam lithography can provide the small scale feature resolution. To be able to rapidly tune the geometries, we have chosen to use electron beam lithography and use PMMA A4 (with a molecular weight of $\sim 950 \mathrm{~K}$ ) to achieve clean liftoff while still meeting the required resolution. A $50 \mathrm{~nm}$ thick alumina mask is sputter coated with a Temescal TES BJD-1800 DC reactive sputter deposition system by depositing aluminum in the presence of oxygen plasma for 5 minutes. The room temperature silicon plasma etch recipe for nanoscale features is described by Henry et al. [15]. The etch recipe was iteratively optimized to achieve uniform etch depth for different pillar widths and uniform sidewall roughness. Etching results are shown in Figure 4, demonstrating uniformity of etch over different pillar sizes and over large arrays.

We then thermally oxidized the pillar structures in a wafer furnace at $1000^{\circ} \mathrm{C}$ for 90 minutes followed by 15 -minute nitrogen anneal with a gradual return to room temperature. The results show a very uniform and continuous layer of oxide as seen in Figure 5. A FEI Sirion 200 scanning electron microscope was used for this high contrast imaging. The Si core and the oxide outer layer can be differentiated due to the second electron emission imaging contrast between silicon and its oxide.

For standard CMOS devices, thick top metal $(\sim 4.6 \mu \mathrm{m})$ aluminum is used for laying out the sensor electrodes 


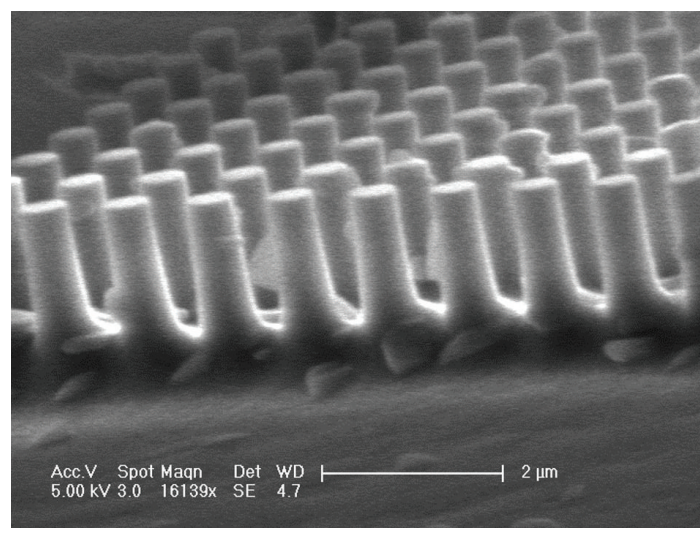

FIGURE 6: Nanopillars etched in CMOS top metal pads.

integrated in the CMOS process itself. This aluminum was patterned using a combination of wet and dry processing techniques. First, ma-N 2400 resist was used as either an electron beam or deep UV resist based upon feature size. A dilute TMAH based developer (e.g., MF319) was used for resist development which also results in some etching of underlying aluminum. This was followed by a Unaxis RIE based system utilizing a mixture of boron trichloride $\left(\mathrm{BCl}_{3}\right)$ and chlorine $\left(\mathrm{Cl}_{2}\right)$ plasma for etching rest of aluminum. This process resulted in very uniform pillar arrays of aluminum, as shown in Figure 6.

Both silicon and aluminum are not very suitable for electrochemical sensing directly [23]. Hence, the top materials were chosen to be more suitable materials depending upon the particular application. For this purpose, sputter deposition of low-impedance metals was used to achieve conformal coatings of Pt group metals. High density argon plasma of 20 millitorr was used to increase the isotropy of the deposition. A $10 \mathrm{~nm}$ Ti adhesion layer was first DC sputter deposited followed by $100 \mathrm{~nm} \mathrm{Au}$ or Pt films, which were DC sputtered onto the Ti. The deposited film layer grew a conformal coating with uniform thickness on the top, side, and base of both pillars and substrate as shown in Figure 7.

In our designs, the $\mathrm{RE}$ consists of a planar $\mathrm{Ag} / \mathrm{AgCl}$ electrode. The $\mathrm{Ag} / \mathrm{AgCl}$ bilayer is formed by vacuum deposition of $300 \mathrm{~nm}$ of $\mathrm{Ag}$ on the RE using liftoff. This is followed by low-power RIE chlorine plasma (10 W forward power) to convert a thin $(100 \mathrm{~nm})$ top layer into $\mathrm{AgCl}$. The composition of the film is confirmed using SEM and EDX analysis as well as by a change in color of the electrode surface (from the white "silver" color to a brown $\mathrm{AgCl}$ color). SEM images of $\mathrm{Ag}$ and $\mathrm{AgCl}$ films on our electrodes are shown in Figure 8.

The completed electrochemical cell needs to be encapsulated in a material which isolates it from its liquid environment and leaves only the sensor exposed to the fluids to be measured. This also enables the formation of a relief well structure to hold the functionalization chemistry in place close to the sensor contact. We used a thin layer of lithographically patterned SU8 (approximately $2 \mu \mathrm{m}$ ) as the insulator/passivation layer, as shown in Figure 9.

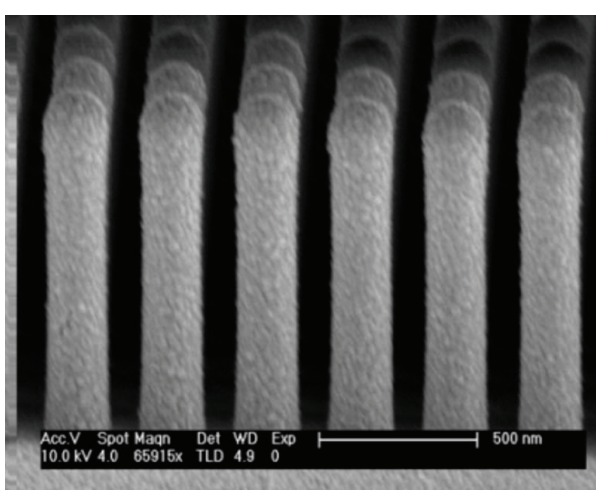

(a)

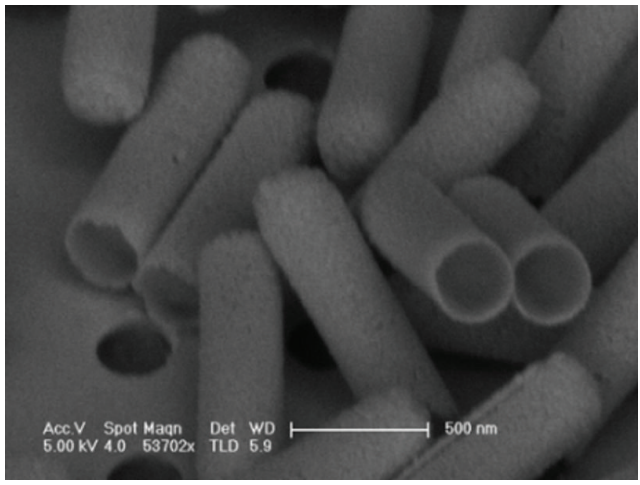

(b)

FIGURE 7: Nanopillars after sputter coating of $50 \mathrm{~nm}$ of $\mathrm{Au}$, (a) pillar array, and (b) pillar array after tweezing to show the metal contacts at the base.

Overall, this lithographic electrochemical cell fabrication process itself confirms that the nanopatterned electrode structure is mechanically robust as it can successfully withstand high surface tension polymer resist application and subsequent processing involving multiple immersions into solvents and water. Individual liquid drop evaporation tests were also performed on these devices and showed no destructive effects. This demonstrated that we can achieve patterned surfaces with simple vacuum-based processing techniques.

\section{Testing and Results}

The sensors were tested to quantify the effects of surface patterning on electrochemical sensing performance and determine if the extra processing incurred for patterning is worth its potential advantages. For all cases, we used a planar sensor as a reference and compared the patterned sensors to the reference for their performance.

Sensors were tested using a commercial CHI 7051D Potentiostat and an electrochemical test cell with a cell stand positioned on a programmable hot plate/stirrer. A small magnetic stirrer was used to allow fast and uniform mixing of test solutions in the background solution. The background solution was $0.01 \mathrm{M}$ PBS ( $\mathrm{pH}$ 7.4) in all cases. A computercontrolled syringe pump (NE-300) was used to introduce 


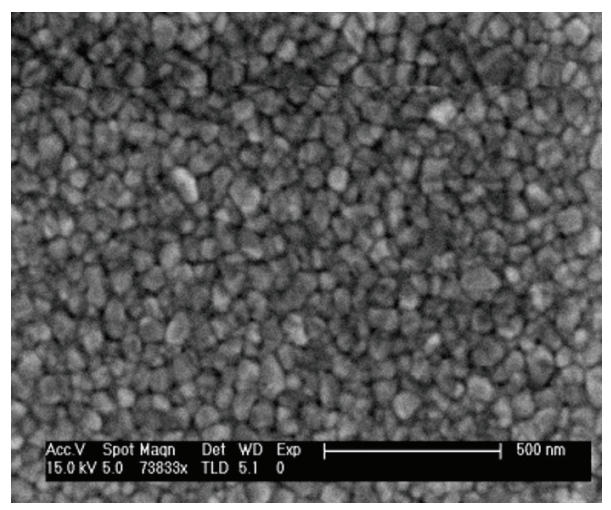

(a)

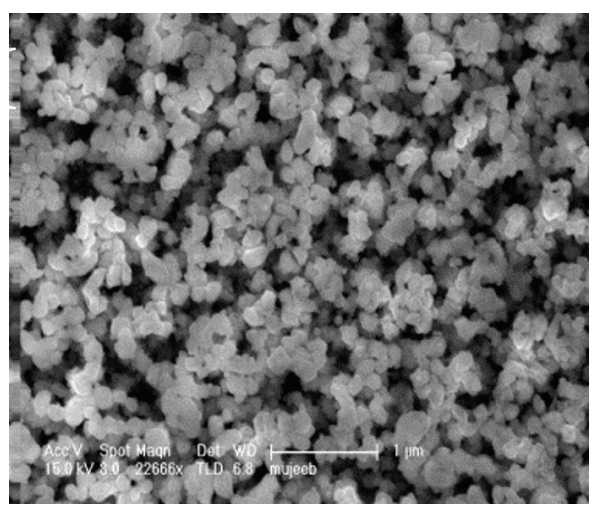

(b)

Figure 8: Thin film reference electrode materials, (a) Ag and (b) $\mathrm{AgCl}$.

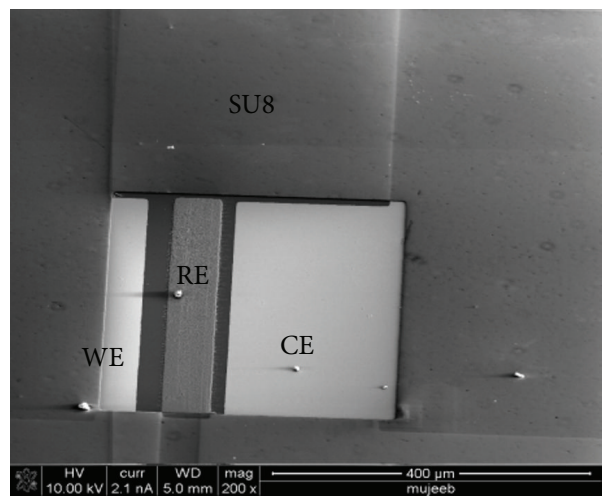

(a)

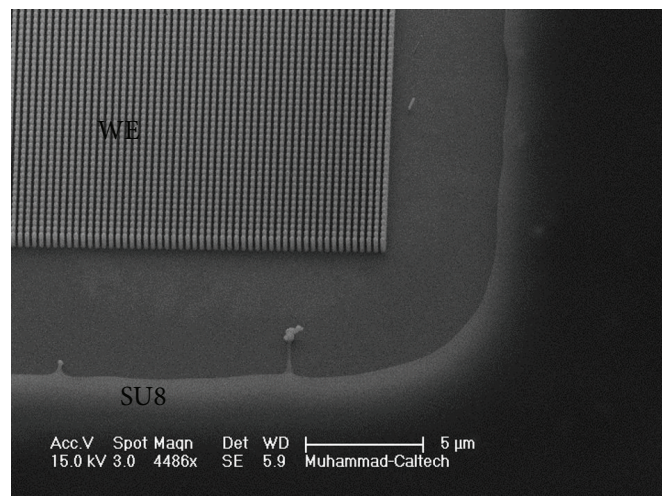

(b)

FIGURE 9: Polymer (SU8) encapsulation around sensors, (a) encapsulation around three-electrode sensor, and (b) higher resolution view showing pillars with SU8 encapsulation surrounding.

small volumes of test solutions in a slow flow-cell. A depiction of the experimental setup is shown in Figure 10.

Results of different electrochemical experiments performed on the fabricated devices are summarized in following subsections.

5.1. Electrochemical Impedance Spectroscopy. Electrochemical impedance spectroscopy (EIS) is used to evaluate the impedance of an electrochemical cell under a given set of test parameters. A small AC signal is applied to the electrochemical cell on top of a DC bias potential and resulting AC current is measured using the Potentiostat. To understand the expected results from these measurements, we used the Randles model of an electrochemical cell which includes the impedance of the electrodes and the solution modeled as a combination of impedances [16], as illustrated in Figure 11.

In this model, $C_{\mathrm{dl}}$ is the double layer capacitance at the electrode-electrolyte interface, $R_{\mathrm{ct}}$ is the contact (charge transfer) resistance between the electrode and the electrolyte, $Z_{w}$ is the diffusion limitation (Warburg impedance) from the bulk to the electrode-electrolyte interface, and $R_{s}$ is the series resistance of the bulk solution between the electrodes.
Analytically, the electrochemical parameters of the system depend upon electrode area and the frequency of operation. For example, the double layer capacitance and contact resistance for an electrode with surface area $A$ are given by the following equations [16]:

$$
\begin{aligned}
& C_{\mathrm{dl}}=K * A, \\
& R_{\mathrm{ct}}=\frac{k T}{z q}\left|\frac{1}{i_{T}}\right| .
\end{aligned}
$$

A typical value of $K$ is $10-100 \mu \mathrm{F} / \mathrm{cm}^{2}$ for most materials and it is different for different materials. $k$ is the Boltzmann constant, $T$ is temperature (Kelvin), $z$ is the ionic charge, $q$ is the electronic charge, and $i_{T}$ is the electrode current. The solution resistance can be calculated from its resistivity $(\rho)$ and electrode spacing $(L)$ and overlapping area $(A)[16]$ :

$$
R_{s}=\frac{\rho L}{A} .
$$

For fast voltage changes (e.g., chronometry or cyclic voltammetry), current is given by the Cottrell equation, as a function 


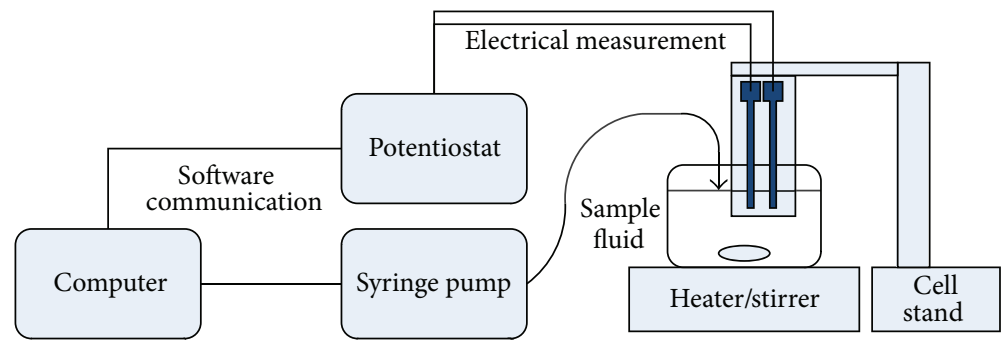

FIgURE 10: Schematic of the electrochemical cell test setup.

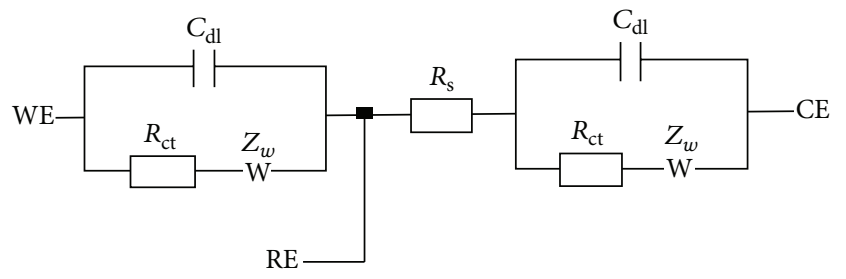

FIGURE 11: Randles equivalent electrical model of an electrochemical cell.

of time and diffusion characteristics of the specie(s) of interest [16]:

$$
I=n F A c_{o} \sqrt{\frac{D}{\pi t}} .
$$

Here, $F$ is faraday constant, $D$ is the diffusion coefficient, $n$ is the number of electrons involved in the reaction, $C_{o}$ is the initial concentration of the specie, and $t$ is time. If we model nanopatterned electrodes simply as planar electrodes of the same total geometric area and ignore the overlap in electric double layer (using considerably large \{greater than $1 \mathrm{~nm}$ \} spacing between the nanopillars), then we can apply the above equations that show that the current is proportional to the electrode surface area and hence the contact resistance decreases proportionally with increase in electrochemical surface area due to the patterning. In addition, the double layer capacitance increases proportionally and the solution resistance also decreases proportionally to the surface area. The CE is designed to be an order of magnitude larger that the WE so that it does not limit the WE output. Hence, the cell impedance would be determined by the WE impedance for practical applications. For simplicity, neglecting the Warburg impedance (diffusion limitations) and assuming that the double layer capacitance acts as a simple capacitor, the cell impedance is given by the parameters of the WE [16], as

$$
Z_{\text {cell }}=\frac{1}{1 / R_{\mathrm{ct}}+j 2 \pi f C_{\mathrm{dl}}}+R_{s} .
$$

Here, $f$ is the frequency of operation in Hertz. Since both resistors are inversely proportional to the surface area and the capacitance is proportional to the surface area, the above equation shows that the cell impedance is inversely proportional to the surface area of the working electrodes. Also, the solution resistance (typically in $\mathrm{K} \Omega$ range) is generally much smaller than electrode resistance (typically in $\mathrm{M} \Omega$ range) for the electrode sizes used in this work and can be neglected. In such cases, (9) can be further simplified to

$$
Z_{\text {cell }}=\frac{R_{\mathrm{ct}}}{1+j 2 \pi f C_{\mathrm{dl}} R_{\mathrm{ct}}} .
$$

Equation (10) is a simple electrical equivalent impedance of a parallel RC network. However, in EIS experiments, frequency $(f)$ is also a control variable and is not fixed. This shows that the cell impedance is dependent upon electrode parameters as well as on the frequency of operation. The frequency of operation depends upon the actual use case but is mostly near DC for most biosensor applications [24].

In our experiments, the frequency of the EIS input $\mathrm{AC}$ signal was swept from $100 \mathrm{KHz}$ to $1 \mathrm{mHz}$ with a small bias of $10 \mathrm{mV}$ using the CHI 7051D Potentiostat. Sensors with different working electrodes and varying pillar sizes $(10 \mu \mathrm{m}$, $300 \mathrm{~nm}$, and $200 \mathrm{~nm}$ ), but with the same Pt counter electrode and $\mathrm{Ag} / \mathrm{AgCl}$ reference electrode, were tested. A simple planar electrode was also tested for reference purposes.

The result of fitting the EIS data for the different cells to the Randles model gives us numerical values for the different components of the cell. Some experimental results of impedance spectroscopy on patterned electrodes are shown in the Nyquist plot format in Figure 12.

These results clearly show that as the pillar diameter is reduced (and hence the number of pillars is increased) the real and imaginary components of the impedance are both reduced. It is also evident that the cell impedance represents an RC circuit as the imaginary component of impedance is negative. The decrease in impedance with frequency can be better seen through a Bode plot, as shown in Figure 13.

This plot shows that the difference between electrode impedances is in fact more pronounced at lower frequencies. This is suitable for most biochemical sensing applications as the methods of detection in such cases are based upon relatively slow voltage changes (e.g., constant potential amperometry, cyclic voltammetry). This can also be used for electrophysiological applications where smaller electrode sizes are necessary but high electrode impedance is problematic for quality of measurements [25].

From Figure 13, a decrease in impedance from downscaling nanostructures is expectable, but the measured decrease is surprisingly more than an order of magnitude in all cases and at $200 \mathrm{~nm}$ spacing, which reaches more than two orders of magnitude decrease. This is larger than expected from 


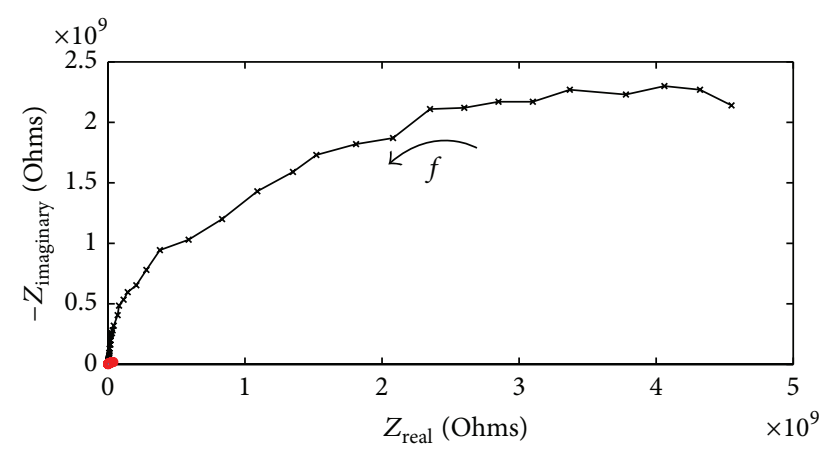

(a)

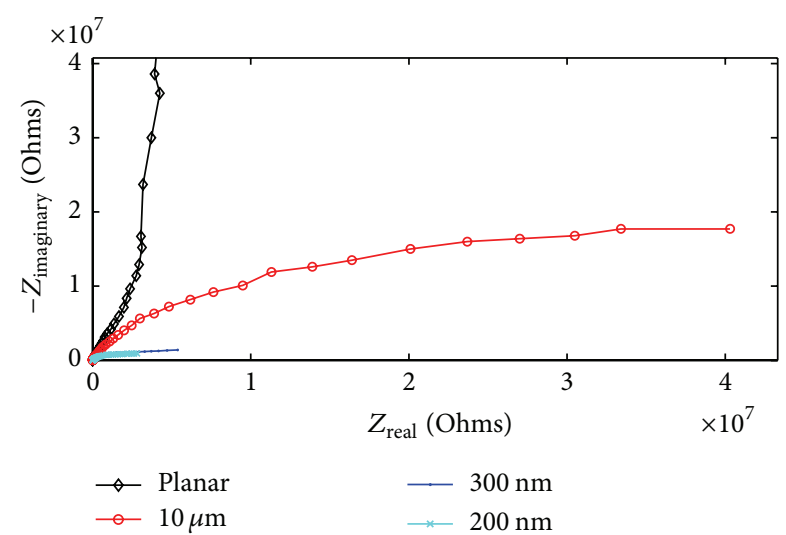

(b)

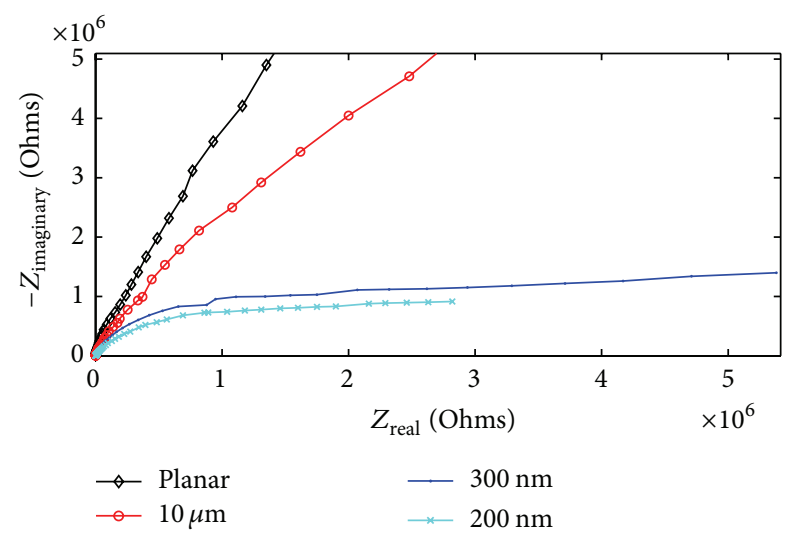

(c)

FIGURE 12: Electrochemical impedance spectroscopy results: (a) comparison of planar and patterned electrodes $(10 \mu \mathrm{m}, 300 \mathrm{~nm}$, and $200 \mathrm{~nm}$ ) on the same scale. Direction of arrow indicates increasing frequency. (b) Rescaled view of impedances to highlight micropatterned electrodes. (c) Rescaled view of impedances to highlight nanopatterned electrodes.

a purely geometric surface area increase, as predicted by (2) $(S / S o=21)$. We speculate that this anomaly results from the nondiffusion limited design of the micro/nanoelectrodes and higher charge transfer capabilities of such smaller electrodes. There is some similarity between our results and other related works reporting higher charge transfer efficiencies to nanopatterned surfaces [10].

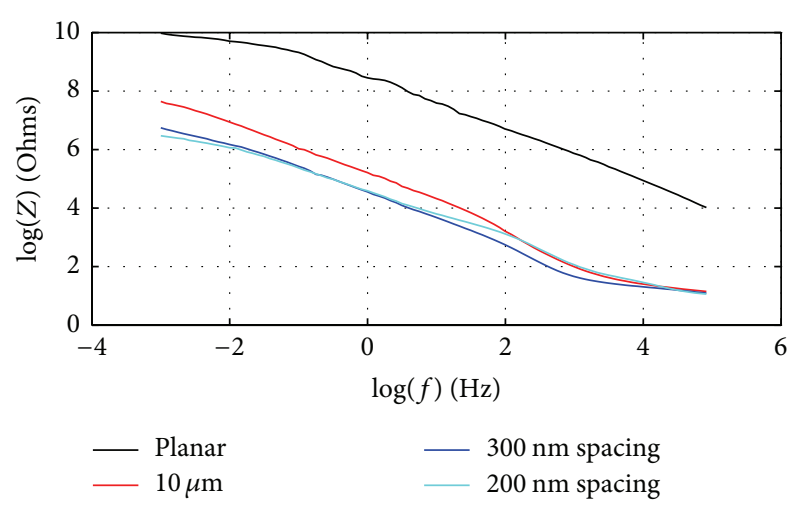

FIGURE 13: Bode plot of electrode impedance (on $\log / \log$ scale).

The relation between decreasing impedance and smaller nanostructuring will end once the electric double layer of adjacent nanostructures begins to overlap and then the enhancement in overall effective surface area (electrochemical equivalent surface area) will become lower than the actual device surface area. This interference happens when the nanopillar spacing is comparable to the electric double layer thickness, which itself is dependent upon the electrolyte in the relevant environment. However for the common electrolytes the electric double layer should only range from about $0.1 \mathrm{~nm}$ to $1 \mathrm{~nm}$ [26], so there is currently little concern about this limitation.

5.2. Nucleic Acid Sensing. Nucleic acid (e.g., DNA) sensing has many biomedical applications including disease diagnostics and gene mutation detection [27]. Electrochemical DNA sensors are capable of sensitive and selective detection of DNA strands and mutations through binding (hybridization) reactions using different detection mechanisms [28]. Most methods of DNA sensing work by attaching ssDNA strand to an electrode as a probe. The probe can have a redox molecule attached to it and its resulting redox current can provide an indication of the configuration of the probe strand. When a complimentary (target) ssDNA is introduced to the solution, it binds with the probe and changes the configuration of the DNA strand. This change moves the redox molecule further away from the surface of the electrode than its resting position thus causing a change in redox current at the electrode [29]. There are different redox species that can be used for this purpose including methylene blue $(\mathrm{MB})$ and ferrocene $(\mathrm{Fc})$ [30]. We chose MB as an indicator for DNA hybridization as it has been widely reported to have a good conjugation efficiency with DNA and can create a significant signal change in its different states [29]. It has been shown to be effective in many cases of hybridization based sensors, for example, for detection of pathogen DNA [31] and proteins using aptamer [32].

Our probe DNA consists of 17 bases and has a MB redox molecule attached at its $5^{\prime}$ end and a C6 thiol at its $3^{\prime}$ end. The target DNA is a complimentary strand with 17 bases. We used gold working electrodes $(100 \mathrm{~nm}$ sputtered gold layer) for these sensors due to ease of thiol bonding between 
the nucleic acid strands and electrode surface. The CE was covered with $100 \mathrm{~nm}$ Pt and the $\mathrm{RE}$ was an $\mathrm{Ag} / \mathrm{AgCl}$ bilayer planar electrode. The WE and CE were patterned with $250 \mathrm{~nm}$ diameter pillars with $500 \mathrm{~nm}$ spacing between pillars. For experimental protocol, we followed the approach previously demonstrated by Rowe et al. [33]. The probe and target DNA was purchased from Biosearch Technologies, Inc., in dry powder form. The probe DNA was dissolved in PBS solution $(\mathrm{pH} 7.4,0.01 \mathrm{M})$ and its concentration was measured using a Nanodrop 2000c spectrophotometer. A typical concentration of $2 \mu \mathrm{M}$ of the DNA stock solution was prepared. Tris(2carboxyethyl) phosphine (TCEP) solution was also mixed in PBS to achieve $50 \mathrm{mM}$ concentration and was added into the DNA stock solution (in 2:1 volume ratio between DNA and TCEP solution) to reduce any disulfide bonds in the DNA solution. The solution was left at room temperature for 20 minutes to complete this reduction. The DNA stock solution was then diluted with PBS to achieve appropriate probe concentrations (typically $100 \mathrm{nM}$ ) and the sensors were immersed in this solution for three hours. Next, the sensors were immersed in a $2 \mathrm{mM}$ mercaptohexanol solution in PBS for six hours to form a back-filling self-assembled monolayer (SAM) to minimize the formation of direct bonds between any target DNA and the gold electrodes. Finally, these electrodes were used in the standard electrochemical test setup consisting of a beaker on a cell stand and connection to the Potentiostat. A background signal was collected by running square wave voltammetry from $0 \mathrm{~V}$ to $-0.6 \mathrm{~V}$ at $100 \mathrm{~Hz}$ versus the $\mathrm{Ag} / \mathrm{AgCl}$ reference electrode. Target DNA solution was made by dissolving the DNA powder in PBS, measuring the resulting concentration optically (using the Nanodrop 2000c spectrophotometer) and then appropriately diluting it to reach a stock concentration of $2 \mu \mathrm{M}$. Controlled amounts $(100 \mu \mathrm{L})$ of this target DNA solution were then added to the background PBS solution (using a syringe pump) to provide an overall target concentration of $10 \mathrm{nM}$ in the final solution. The same voltammetry cycle was repeated on various time intervals. The difference in the peak current before and after the addition of the target DNA corresponds to the decrease in redox current of the methylene blue probe as a result of the change in the morphology of the probe strand due to hybridization. We measured the time dependence of the hybridization process and confirmed excellent sensor sensitivity towards the target DNA. The results for nanopatterned electrode are shown in Figure 14.

The results indicate that surface nanopatterning increases the signal level by decreasing the overall electrochemical impedance and by increasing the hybridization efficiency with larger number of hybridization target sites. As a false positive test, the experiment was repeated with nonspecific target DNA, confirming that there is no appreciable hybridization or binding in that case.

The measured electrical current levels for the patterned sensors are comparable to those measured in a macroscale planar electrode ( $3 \mathrm{~mm}$ diameter Au electrode) which we used as a reference in this study. The following graph (Figure 15) compares the response of a nanopatterned sensor with the planar sensor. It shows that nanopatterned sensor provides orders of magnitude more responsive compared
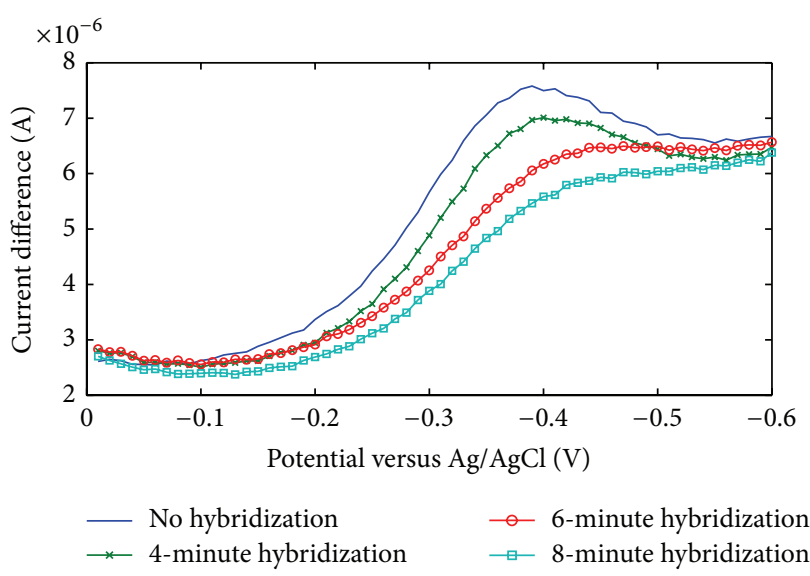

FIGURE 14: Square wave voltammetric detection of DNA hybridization.

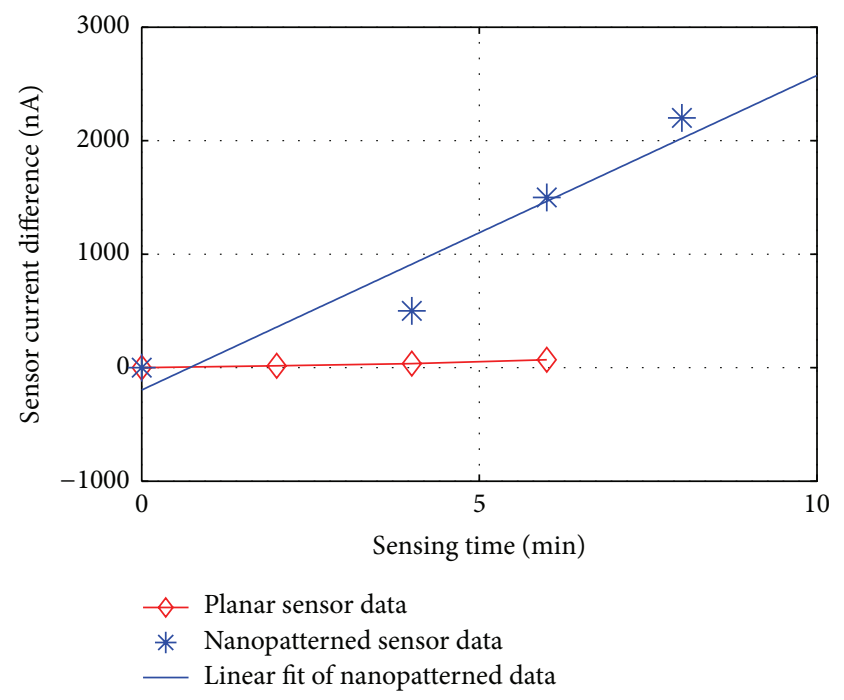

FIgURE 15: Comparison of nucleic acid hybridization detection efficiency for planar and nanopatterned sensors.

to the planar sensor and that actual enhancement is more than that just predicted by the increase in surface area, as per (2). We speculate that this is similar to the decrease in electrode impedance as in the EIS experiments described earlier. We suggest that the mechanism of increase in signal is both due to the increase in electrode surface area and a change in the diffusion profile of nucleic acid molecules (3D near a nanopatterned surface versus $2 \mathrm{D}$ for a planar surface) and increase in charge transfer efficiency due to the nanopatterning.

These results demonstrate that nanopatterned electrodes can be effectively used for nucleic acid sensing applications where available area is environmentally constrained. Some example applications are system-on-chip devices, point of care diagnostic devices, and long-term implants using aptamers [34]. 


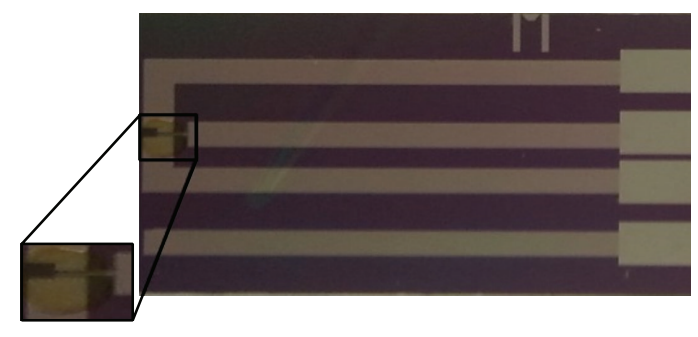

FIgURE 16: Glucose sensor after functionalization.

5.3. Glucose Sensing. Glucose sensing is an important application for electrochemical sensors since it can provide clinically valuable and accurate results for millions of diabetic patients [35]. We fabricated sensors based upon platinum working electrodes for glucose measurements. The choice of material was based upon high electrochemical activity of platinum for metabolic sensing applications [36]. The CE was also $\mathrm{Pt}$ and the $\mathrm{RE}$ was made of $\mathrm{Ag} / \mathrm{AgCl}$. The electrodes were functionalized with glucose oxidase enzyme to achieve a glucose selective response as it is the consensus standard enzyme for electrochemical glucose sensors [37]. The immobilization was performed in situ using BSA based hydrogel as the immobilization matrix. The required solutions were made in $0.01 \mathrm{M}$ PBS with a $\mathrm{pH}$ close to that of human body fluids (7.4). The hydrogel used was based upon Bovine Serum Albumin (BSA) solution and was used to immobilize glucose oxidase on the electrodes. Glutaraldehyde was used as a crosslinking agent for the hydrogel.

The experimental procedure for immobilization was adopted from the literature and iteratively optimized for our application [38]. Glucose oxidase (GOx) solution was made by dissolving $1.6 \mathrm{mg}$ of GOx (Sigma-Aldrich type VII from Aspergillus niger) powder in $10 \mu \mathrm{L}$ of PBS. The Nanodrop 2000c was used to confirm the concentrations of enzyme in the resulting solution. BSA solution was made in PBS by dissolving $4 \mathrm{mg}$ powder in $30 \mu \mathrm{l}$ of PBS. Glutaraldehyde (25\% stock solution) was diluted in PBS to $2.5 \%$. The enzyme solution was first diluted in PBS by adding 1 part of enzyme solution in 4 parts of PBS. The resulting mixture was mixed with BSA solution PBS in 1:1 ratio by volume. Finally, Glutaraldehyde solution was mixed in this mixture by $3: 1$ volume ratio between BSA and enzyme solution and Glutaraldehyde. Then, $1 \mu \mathrm{L}$ solution from the final mixture was pipetted carefully onto the electrochemical sensor. The device was left at room temperature for 10 minutes to allow gel formation while the process was continually observed under a microscope. The sensor was then soaked in PBS for 3 hours to let any unbound enzyme and BSA dissolve away and result in stable gel chemistry. The sensor after functionalization is shown in Figure 16.

The sensor was then dried and placed in the experimental setup with the Potentiostat. Controlled amounts of glucose stock solution $(1 \mathrm{M})$ were added to the background PBS in the electrochemical cell and the resulting signals were measured using the Potentiostat with Cyclic Voltammetry (CV) and amperometry. A typical CV scan is performed using voltage

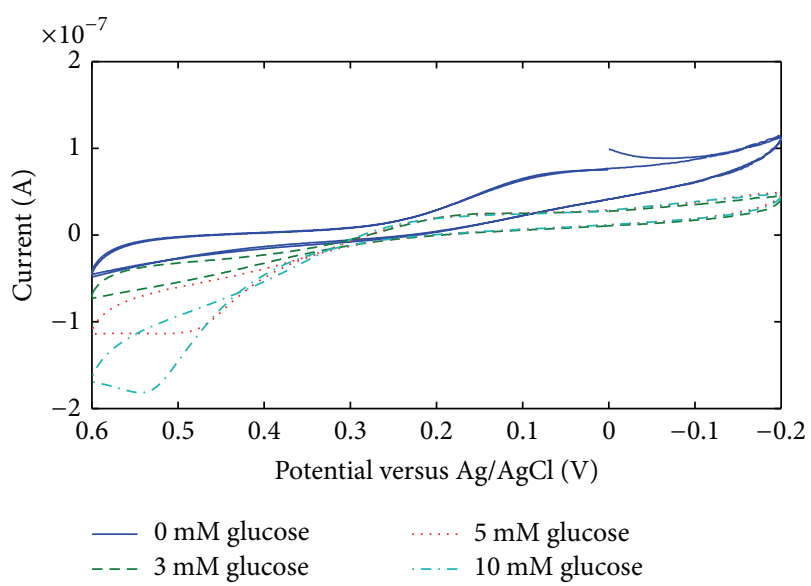

FIGURE 17: In vitro cyclic voltammetric sensing of glucose.

from 0 to $0.6 \mathrm{~V}$ versus the $\mathrm{Ag} / \mathrm{AgCl}$ electrode at a scan rate of $0.01 \mathrm{~V} /$ second. A typical result is shown in Figure 17.

The sensor was fabricated with planar electrodes as well as patterned electrodes $(200 \mathrm{~nm}$ pillar diameter, $500 \mathrm{~nm}$ pillar spacing). The response of a nanostructured sensor with the same electrode geometry as a planar electrode was much higher, as shown in Figure 18. Further enhancement is possible by performing direct immobilization of enzymes on the electrodes which enhances the electron transfer efficiency from the enzyme to the electrode [39].

The glucose sensor response shows saturation effects due to mismatch between glucose and oxygen levels in the solution [40]. The response can be optimized by adding more layers of suitable membranes to the sensor chemistry stack [40] and is the focus of our future efforts. To test specificity, different solutions of PBS and $\mathrm{pH}$ buffers (with $\mathrm{pH} \sim 7$ ) were added as controls but did not cause any appreciable change in the sensor response. Overall, the sensitivity increase due to nanopatterning was more than two orders of magnitude as compared to the planar electrode. This response is similar to what we observed in the earlier described EIS and nucleic acid sensing and likely follows from the same geometric and diffusion reasons.

These results indicate that the miniaturized sensors can provide good sensitivity towards glucose in the physiological range $(2-20 \mathrm{mM})$. Such sensors, when combined with appropriate circuitry, wireless powering, and telemetry techniques, can form the basis of ultra-small (mm scale) size and costeffective integrated sensing platforms [41]. The resulting small size can pave the way for such systems to be used for long-term in vivo applications [42], thus revolutionizing the field of continuous glucose monitoring and other similar sensing applications.

\section{Summary and Conclusions}

In this paper, we demonstrated the advantages of integrated electrochemical sensors based upon micro/nanopatterned electrodes over traditional planar sensors. We showed that when the die area is limited, such patterning techniques 


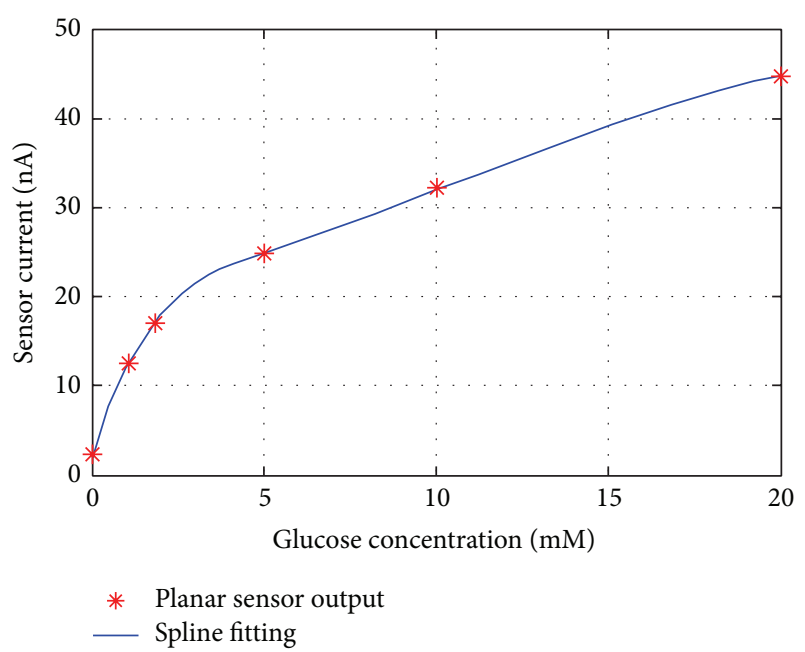

(a)

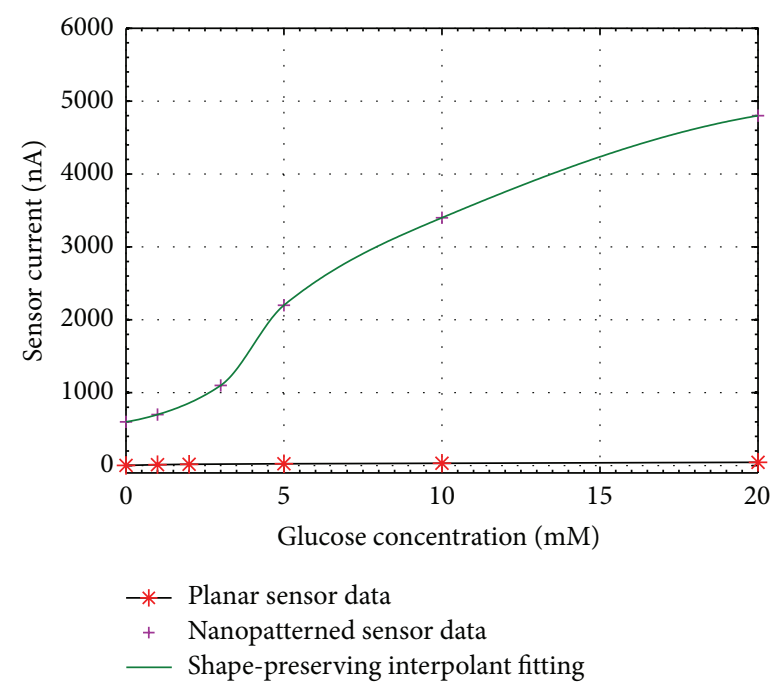

(b)

Figure 18: Response curve of glucose sensor. (a) Planar sensor. (b) Patterned sensor.

can overcome the sensitivity limitations of electrochemical sensors. Hence, adequate signal to noise ratio can be achieved in different applications resulting in miniaturization of overall sensing platforms. We also demonstrate that such patterning can be achieved using standard fabrication techniques, thus limiting the need of special methods which can add to cost and reliability of the fabrication process. This has the advantage that such electrodes can be created using common fabrication facilities with currently running infrastructure which allows for low device fabrication costs and high manufacturing reproducibility. Such patterning aids in the goal of shrinking electrode sizes due to measurement environment constraints and allows for smaller total platform footprints. We demonstrated that these methods can be used with standard substrates (e.g., $\mathrm{Si}$ ) as well as metal electrodes on CMOS substrates but other material systems may also be applicable.
We validated the effect of surface patterning in decreasing electrode impedance for a given electrode size. We showed that the smaller the scale of patterning is, the denser the resulting patterns are and hence the more pronounced is the enhancement in electrode properties (decrease in impedance). We also provided examples of DNA and glucose sensing to demonstrate the sensitivity advantage of such patterned electrodes but broader applications are possible such as in electrophysiology. Furthermore, we argue that using such techniques on standard electronic platforms (like CMOS) is very favorable for scaling production of a sensing platform using integrated CMOS-MEMS facilities which are becoming more and more cost effective with time [43]. This would minimize the failure modes related with production of large number of sensors using prior methods which are more difficult to scale and need more specialized facilities for proper manufacturing control.

Our future work is focused on broadening the range of applications for such electrodes as well as in optimizing the sensor chemistry to result in more efficient sensors for realworld applications.

\section{Appendix}

(a) For patterned electrode with hexagonal arrangement of pillars with height $h$, radius $r$, and separation $a$, surface area enhancement is due to the area of pillars which is a product of their circumference and height. Hence, total surface area for the patterned electrode is sum of the rectangular geometric area and the circumferential surface area. The surface area for such hexagonal packed structure can be calculated using a unit cell, as described here [10].

The surface area of planar electrode is given by

$$
\text { So }=\frac{3 \sqrt{3}}{2} a^{2} \text {. }
$$

The surface area of patterned electrode is given by

$$
S=\frac{3 \sqrt{3}}{2} a^{2}+6 \pi r h
$$

Hence, the area ratio would be given by

$$
\frac{S}{S o}=1+\frac{4 \pi r h}{\sqrt{3}} \frac{1}{a^{2}}=1+7.26\left(\frac{r}{a}\right)\left(\frac{h}{a}\right) .
$$

(b) For rectangular electrode, surface area of planar electrode is given by (assuming length $L$ of both sides)

$$
\text { So }=L^{2} \text {. }
$$

For patterned electrode with rectangular arrangement of pillars with height $h$, radius $r$, and separation $a$, surface area enhancement is due to the area of pillars which is a product of their circumference and height. The circumferential area depends upon number of pillars which is given by ratio of each side to the pillar separation (center to center distance):

$$
S=L^{2}+2 \pi r h \frac{L^{2}}{a^{2}} \text {. }
$$


Hence, the area ratio would be given by

$$
\frac{S}{S o}=1+2 \pi r h \frac{1}{a^{2}}=1+6.28\left(\frac{r}{a}\right)\left(\frac{h}{a}\right) .
$$

\section{Conflict of Interests}

The authors declare that there is no conflict of interests regarding the publication of this paper.

\section{Acknowledgments}

The fabrication procedures in this work were performed at the Kavli Nanoscience Institute at the California Institute of Technology. This work was supported by Sanofi A. G. under Grant no. D09/4502653189. The authors would like to thank Mr. Mehmet Sencan for his help with functionalization and testing.

\section{References}

[1] C. M. Li, H. Dong, X. Cao, J. Luong, and X. Zhang, Implantable Electrochemical Sensors for Biomedical and Clinical Applications: Progress, Problems, and Future Possibilities, vol. 14, Bentham Science Publishers, 2007.

[2] S. Ayers, K. D. Gillis, M. Lindau, and B. A. Minch, "Design of a CMOS potentiostat circuit for electrochemical detector arrays," IEEE Transactions on Circuits and Systems I: Regular Papers, vol. 54, no. 4, pp. 736-744, 2007.

[3] X.-J. Huang and Y.-K. Choi, "Chemical sensors based on nanostructured materials," Sensors and Actuators B: Chemical, vol. 122, no. 2, pp. 659-671, 2007.

[4] E. Jubete, O. A. Loaiza, E. Ochoteco, J. A. Pomposo, H. Grande, and J. Rodríguez, "Nanotechnology: a tool for improved performance on electrochemical screen-printed (bio)sensors," Journal of Sensors, vol. 2009, Article ID 842575, 13 pages, 2009.

[5] A. K. Wanekaya, W. Chen, N. V. Myung, and A. Mulchandani, "Nanowire-based electrochemical biosensors," Electroanalysis, vol. 18, no. 6, pp. 533-550, 2006.

[6] J. H. T. Luong, R. S. Brown, and W. H. Scouten, "Enzyme or protein immobilization techniques for applications in biosensor design," Trends in Biotechnology, vol. 13, no. 5, pp. 178-185, 1995.

[7] M. Mujeeb-U-Rahman and A. S. M. H. Nazari, "An implantable continuous glucose monitoring microsystem in $0.18 \mu \mathrm{m}$ CMOS," in Proceedings of the VLSI Syposium, Honolulu, Hawaii, USA, June 2014.

[8] W. K. Ward, E. P. Slobodzian, K. L. Tiekotter, and M. D. Wood, "The effect of microgeometry, implant thickness and polyurethane chemistry on the foreign body response to subcutaneous implants," Biomaterials, vol. 23, no. 21, pp. 4185-4192, 2002.

[9] M. Lee, Y. Jeon, T. Moon, and S. Kim, “Top-down fabrication of fully CMOS-compatible silicon nanowire arrays and their integration into CMOS inverters on plastic," ACS Nano, vol. 5, no. 4, pp. 2629-2636, 2011.

[10] G. Zhang, "Design and fabrication of 3D skyscraper nanostructures and their application as electrodes," in Biosensors, New Perspectives in Biosensors Technology and Applications, $\mathrm{P}$. Andrea Serra, Ed., InTech, 2011.

[11] S. M. U. Ali, T. Aijazi, K. Axelsson, O. Nur, and M. Willander, "Wireless remote monitoring of glucose using a functionalized
$\mathrm{ZnO}$ nanowire arrays based sensor," Sensors, vol. 11, no. 9, pp. 8485-8496, 2011.

[12] A. Huczko, "Template-based synthesis of nanomaterials," Applied Physics A: Materials Science and Processing, vol. 70, no. 4, pp. 365-376, 2000.

[13] Y.-J. Lee, D.-J. Park, J.-Y. Park, and Y. Kim, "Fabrication and optimization of a nanoporous platinum electrode and a nonenzymatic glucose micro-sensor on silicon," Sensors, vol. 8, no. 10, pp. 6154-6164, 2008.

[14] R. C. Barry, Y. Lin, J. Wang, G. Liu, and C. A. Timchalk, "Nanotechnology-based electrochemical sensors for biomonitoring chemical exposures," Journal of Exposure Science and Environmental Epidemiology, vol. 19, no. 1, pp. 1-18, 2009.

[15] M. D. Henry, S. Walavalkar, A. Homyk, and A. Scherer, "Alumina etch masks for fabrication of high-aspect-ratio silicon micropillars and nanopillars," Nanotechnology, vol. 20, no. 25, Article ID 255305, 2009.

[16] A. J. Bard and L. R. Faulkner, Electrochemical Methods: Fundamentals and Applications, John Wiley \& Sons, 2nd edition, 2001.

[17] A. Heidelberg, L. T. Ngo, B. Wu et al., "A generalized description of the elastic properties of nanowires," Nano Letters, vol. 6, no. 6, pp. 1101-1106, 2006.

[18] D. Grieshaber, R. MacKenzie, J. Vörös, and E. Reimhult, "Electrochemical biosensors-sensor principles and architectures," Sensors, vol. 8, no. 3, pp. 1400-1458, 2008.

[19] R. A. Sheldon, "Enzyme immobilization: the quest for optimum performance," Advanced Synthesis and Catalysis, vol. 349, no. 89, pp. 1289-1307, 2007.

[20] S. J. Updike and G. P. Hicks, "The enzyme electrode," Nature, vol. 214, no. 5092, pp. 986-988, 1967.

[21] S. Zimmermann, D. Fienbork, A. W. Flounders, and D. Liepmann, "In-device enzyme immobilization: wafer-level fabrication of an integrated glucose sensor," Sensors and Actuators B: Chemical, vol. 99, no. 1, pp. 163-173, 2004.

[22] P. N. Bartlett and D. J. Caruana, "Electrochemical immobilization of enzymes. Part V. Microelectrodes for the detection of glucose based on glucose oxidase immobilized in a poly(phenol) film," Analyst, vol. 117, no. 8, pp. 1287-1292, 1992.

[23] S. Park, H. Boo, and T. D. Chung, "Electrochemical nonenzymatic glucose sensors," Analytica Chimica Acta, vol. 556, no. 1, pp. 46-57, 2006.

[24] S.-M. Park and J.-S. Yoo, "Electrochemical impedance spectroscopy for better electrochemical measurements," Analytical Chemistry, vol. 75, no. 21, pp. 455A-461A, 2003.

[25] V. S. Polikov, P. A. Tresco, and W. M. Reichert, "Response of brain tissue to chronically implanted neural electrodes," Journal of Neuroscience Methods, vol. 148, no. 1, pp. 1-18, 2005.

[26] S. G. Real, J. R. Vilche, and A. J. Arvia, "The impedance response of electrochemically roughened platinum electrodes. Surface modeling and roughness decay," Journal of Electroanalytical Chemistry, vol. 341, no. 1-2, pp. 181-195, 1992.

[27] T. G. Drummond, M. G. Hill, and J. K. Barton, "Electrochemical DNA sensors," Nature Biotechnology, vol. 21, no. 10, pp. 11921199, 2003.

[28] J. J. Gooding, "Electrochemical DNA hybridization biosensors," Electroanalysis, vol. 14, no. 17, pp. 1149-1156, 2002.

[29] S. O. Kelley, J. K. Barton, N. M. Jackson, and M. G. Hill, "Electrochemistry of methylene blue bound to a DNA-modified electrode," Bioconjugate Chemistry, vol. 8, no. 1, pp. 31-37, 1997.

[30] D. Kang, X. Zuo, R. Yang, F. Xia, K. W. Plaxco, and R. J. White, "Comparing the properties of electrochemical-based DNA 
sensors employing different redox tags," Analytical Chemistry, vol. 81, no. 21, pp. 9109-9113, 2009.

[31] A. Erdem, K. Kerman, B. Meric, U. S. Akarca, and M. Ozsoz, "Novel hybridization indicator methylene blue for the electrochemical detection of short DNA sequences related to the hepatitis B virus," Analytica Chimica Acta, vol. 422, no. 2, pp. 139-149, 2000.

[32] G. S. Bang, S. Cho, and B.-G. Kim, "A novel electrochemical detection method for aptamer biosensors," Biosensors \& Bioelectronics, vol. 21, no. 6, pp. 863-870, 2005.

[33] A. A. Rowe, R. J. White, A. J. Bonham, and K. W. Plaxco, "Fabrication of electrochemical-DNA biosensors for the reagentless detection of nucleic acids, proteins and small molecules," Journal of Visualized Experiments, no. 52, article 2922, 2011.

[34] J. Liu, Z. Cao, and Y. Lu, "Functional nucleic acid sensors," Chemical Reviews, vol. 109, no. 5, pp. 1948-1998, 2009.

[35] S. Vaddiraju, D. J. Burgess, I. Tomazos, F. C. Jain, and F. Papadimitrakopoulos, "Technologies for continuous glucose monitoring: current problems and future promises," Journal of Diabetes Science and Technology, vol. 4, no. 6, pp. 1540-1562, 2010.

[36] G.-H. Wu, X.-H. Song, Y.-F. Wu, X.-M. Chen, F. Luo, and $\mathrm{X}$. Chen, "Non-enzymatic electrochemical glucose sensor based on platinum nanoflowers supported on graphene oxide," Talanta, vol. 105, pp. 379-385, 2013.

[37] S. Ferri, K. Kojima, and K. Sode, "Review of glucose oxidases and glucose dehydrogenases: a bird's eye view of glucose sensing enzymes," Journal of Diabetes Science and Technology, vol. 5, no. 5, pp. 1068-1076, 2011.

[38] N. F. de Rooji, M. Koudelka-Hep, and D. J. Strike, "Activation of rayon/polyester cloth for protein immobilization," in Immobilization of Enzymes and Cells, G. F. Bickerstaff, Ed., vol. 1, pp. 77-82, Humana Press, Totowa, NJ, USA, 1997.

[39] M. V. Pishko, A. C. Michael, and A. Heller, "Amperometric glucose microelectrodes prepared through immobilization of glucose oxidase in redox hydrogels," Analytical Chemistry, vol. 63, no. 20, pp. 2268-2272, 1991.

[40] S. Vaddiraju, A. Legassey, Y. Wang et al., "Design and fabrication of a high-performance electrochemical glucose sensor," Journal of Diabetes Science and Technology, vol. 5, no. 5, pp. 1044-1051, 2011.

[41] M. Mujeeb-U-Rahman, D. Adalian, M. Sencan, and A. Scherer, "Nanofabrication techniques for fully integrated sensing platforms," in Nanotech 2013, pp. 73-76, NSTI, 2013.

[42] J. C. Pickup, F. Hussain, N. D. Evans, and N. Sachedina, "In vivo glucose monitoring: the clinical reality and the promise," Biosensors and Bioelectronics, vol. 20, no. 10, pp. 1897-1902, 2005.

[43] A. Witvrouw, "CMOS-MEMS integration: why, how and what?" in Proceedings of the Annual Conference on CAD (Computer Aided Design) (ICCAD '06), San Jose, Calif, USA, November 2006. 

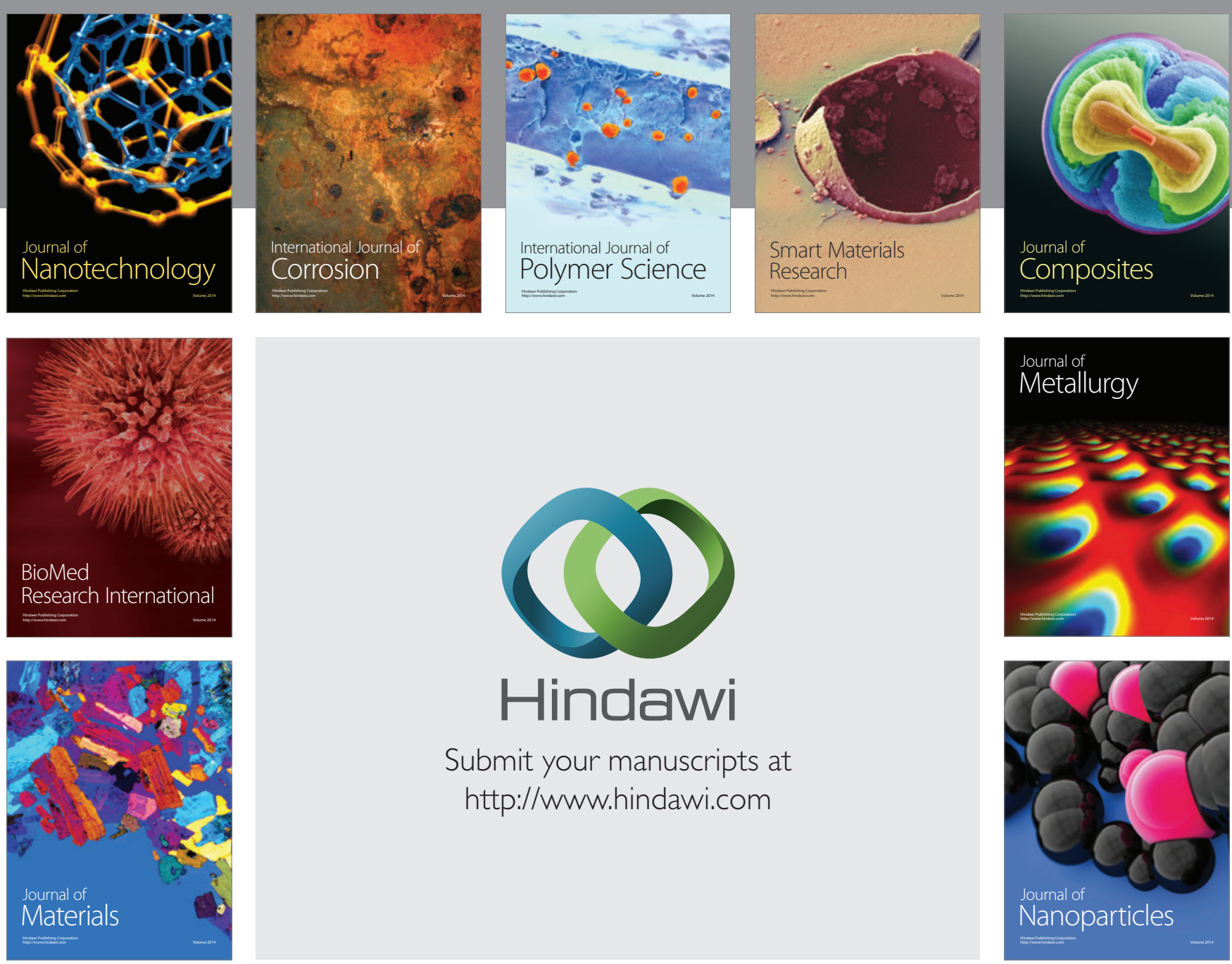

Submit your manuscripts at http://www.hindawi.com
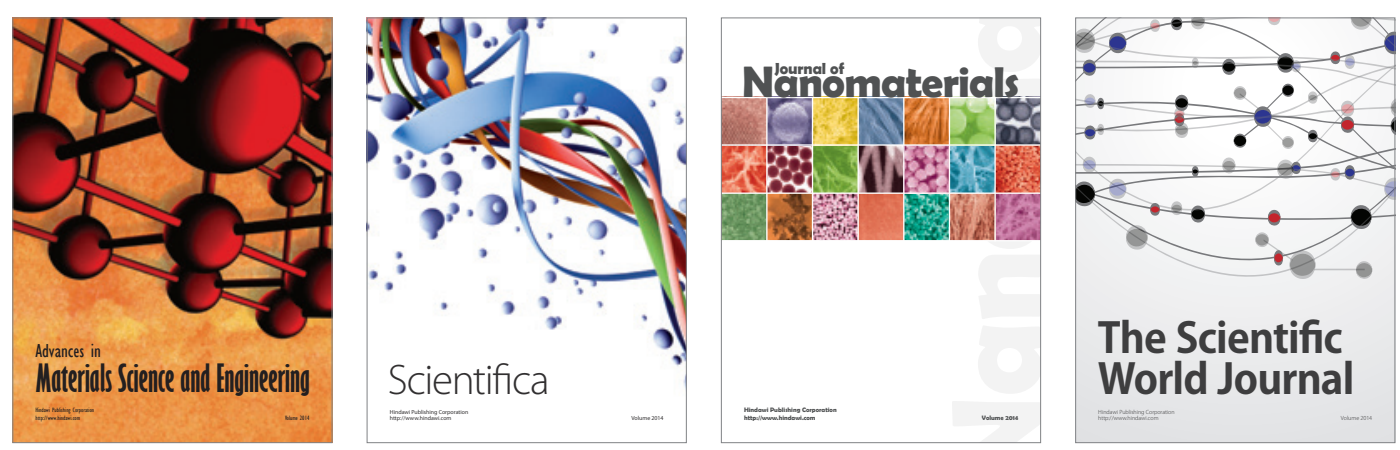

\section{The Scientific World Journal}
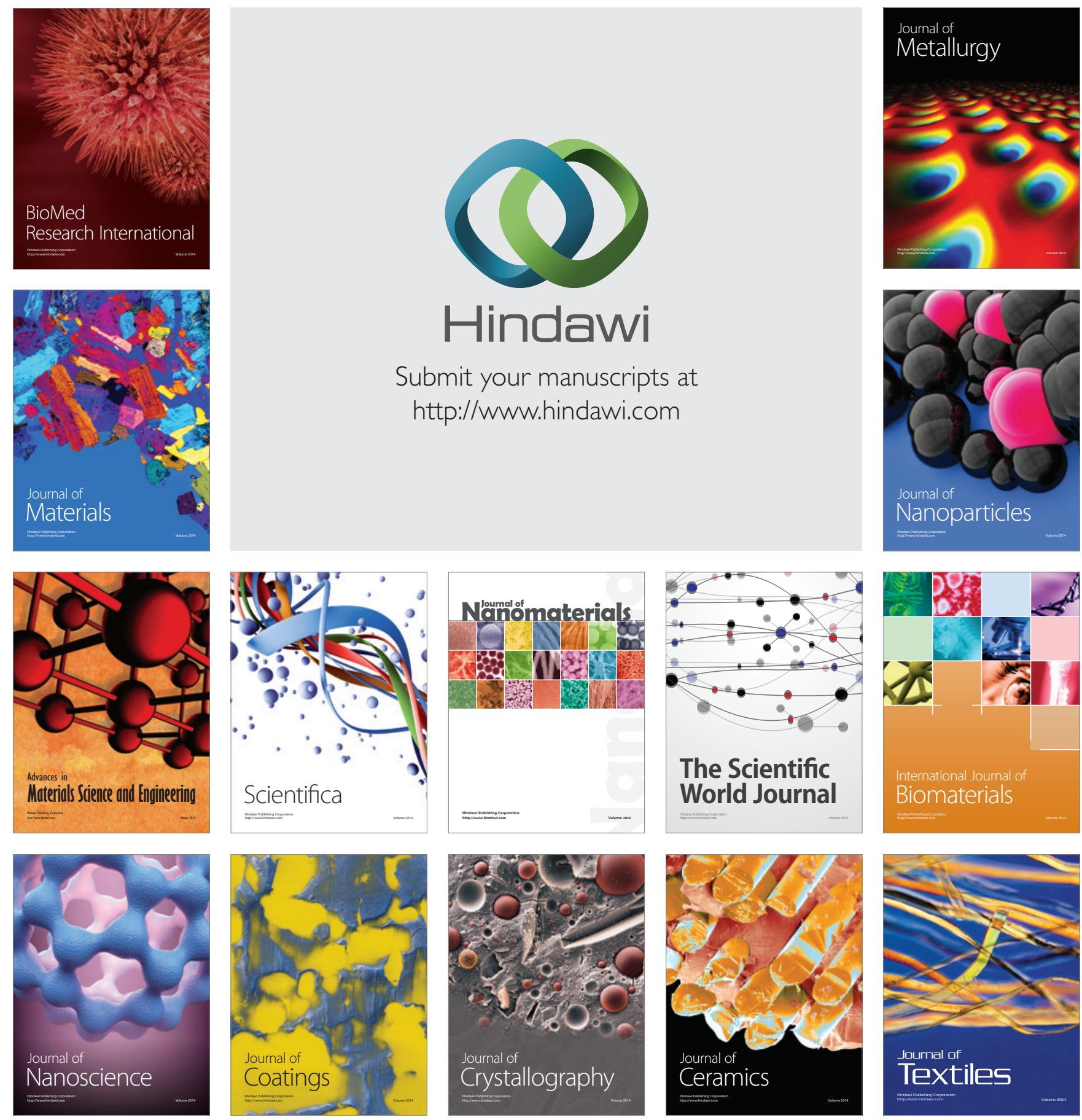